\title{
NMR Detection of Structures in the HIV-1 5'-Leader RNA that Regulate Genome Packaging
}

\author{
Kun Lu ${ }^{1,+, \#, ~ X i a o ~ H e n g ~}{ }^{1,+}$, Lianko Garyu ${ }^{1}$, Sarah Monti ${ }^{1}$, Eric L. Garcia ${ }^{2}$, Siarhei \\ Kharytonchyk $^{2}$, Bilguujin Dorjsuren ${ }^{1}$, Gowry Kulandaivel ${ }^{1}$, Simonne Jones ${ }^{1}$, Atheeth \\ Hiremath ${ }^{1}$, Sai Sachin Divakaruni ${ }^{1}$, Courtney LaCotti ${ }^{1}$, Shawn Barton ${ }^{1}$, Daniel Tummillo ${ }^{1}$, \\ Azra Hosic $^{1}$, Kedy Edme ${ }^{1}$, Sara Albrecht ${ }^{1}$, Alice Telesnitsky ${ }^{2, *}$, and Michael F. Summers ${ }^{1,{ }^{*}}$ \\ ${ }^{1}$ Howard Hughes Medical Institute and Department of Chemistry and Biochemistry, University of \\ Maryland Baltimore County, 1000 Hilltop Circle, Baltimore, Maryland 21250 \\ ${ }^{2}$ Department of Microbiology and Immunology, University of Michigan Medical School, Ann Arbor \\ Ml 48109-0620
}

\begin{abstract}
The 5'-leader of the HIV-1 genome regulates multiple functions during viral replication by mechanisms that have yet to be established. We developed an NMR approach that enabled direct detection of structural elements within the intact leader (712 nucleotide dimer) that are critical for genome packaging. Residues spanning the gag start codon (AUG) form a hairpin in the monomeric leader and base pair with residues of the Unique-5' region (U5) in the dimer. U5:AUG formation promotes dimerization by displacing and exposing a dimer-promoting hairpin, and enhances binding by the nucleocapsid protein (NC), the cognate domain of the viral Gag polyprotein that directs packaging. Our findings support a packaging mechanism in which translation, dimerization, $\mathrm{NC}$ binding, and packaging are regulated by a common RNA structural switch.
\end{abstract}

The 5'-leader is the most conserved region of the HIV-1 genome and is responsible for regulating multiple activities during viral replication, including RNA encapsidation during virus assembly $(1,2)$. Like all retroviruses, HIV-1 packages two copies of its genome, enabling strand-transfer mediated recombination during reverse transcription and promoting genetic evolution under environmental and chemotherapeutic pressure (3). Dimeric genomes are trafficked from the cytoplasm to plasma membrane assembly sites by a small number of viral Gag proteins, where additional Gag proteins assemble and budding occurs (4-8). Dimerization and packaging are mediated by interactions between the nucleocapsid (NC) domains of the viral Gag polyproteins and RNA elements within the 5'-leader of the genome, and there is evidence that these activities and translational control are mechanistically coupled $(7,9-13)$.

Understanding of the mechanisms that regulate 5'-leader activities is limited, due in part to incomplete knowledge of the leader structure (14). Recombinant leader-containing RNAs have been probed by nucleotide accessibility mapping, mutagenesis, and biochemical approaches, and although there is general consensus that transcriptional activation, primer binding, dimerization, and splicing activities are promoted by discrete hairpin structures (10, 15-23) (TAR, PBS, DIS and SD hairpins, respectively; Fig. 1), there is less agreement

\footnotetext{
*Correspondence: ateles@umich.edu; summers@hhmi.umbc.edu.

\#Current address: The RNA Institute, State University of New York at Albany, 1400 Washington Avenue, Albany, NY 12222

${ }^{+}$These authors contributed significantly to this work.
} 
regarding the structures that regulate packaging (14). In particular, residues overlapping the gag start codon (G328-A356, AUG) that are critical for genome packaging (24) and RNA dimer stability (25) have been proposed to form a hairpin (Fig. 1B), to base-pair with residues of the upstream Unique-5' element (U5, Fig. 1C) (10, 20), or to adopt other conformations (14). In vivo nucleotide reactivity mapping has supported multiple AUG models, without consensus $(14,21-23)$. NMR is potentially well suited to probe RNA structure, but signal degeneracy and relaxation problems have thus far limited applications to relatively small oligonucleotides (typically fewer than 50 residues) $(26,27)$. Here we describe an NMR approach that enabled direct detection of structures formed by AUG and other packaging elements within the intact, $230 \mathrm{kDalton}$ dimeric 5'-leader, as well as the identification of conformational changes that regulate dimerization, $\mathrm{NC}$ binding and packaging.

A 356 nucleotide HIV-1 $1_{\mathrm{NL} 4-3} 5^{\prime}$-leader RNA that includes the entire $5^{\prime}$-Untranslated Region ( $5^{\prime}$-UTR) and the first 21 residues of gag $\left(5^{\prime}-\mathrm{L}\right)$ was prepared by enzymatic ligation of nonlabeled 5'-RNA (residues 1-327) and ${ }^{13} \mathrm{C}$-enriched AUG fragments (residues 328-356) (Fig. 1) (28). The 5'-leader exists predominantly as a monomer at low ionic strength (Fig. 1D), and under these conditions the AUG residues of $5^{\prime}-\mathrm{L}$ gave rise to ${ }^{1} \mathrm{H}^{13}{ }^{13} \mathrm{C}$ correlated HMQC (28) NMR spectra similar to those observed for the isolated AUG fragment, which is known to form a hairpin (29) (Fig. 1E,F). NMR line widths of AUG in the intact 5'-leader and the isolated AUG RNA were also similar, indicating that the hairpin is structurally mobile and does not form A-minor-like contacts (30).

Significant changes in the ${ }^{1} \mathrm{H}_{-}{ }^{13} \mathrm{C}$ HMQC spectra of AUG-labeled $5^{\prime}-\mathrm{L}$ were observed upon incubation at physiological ionic strength (PI buffer: $10 \mathrm{mM}$ Tris-HCl, $140 \mathrm{mM} \mathrm{KCl}, 10$ $\mathrm{mM} \mathrm{NaCl}, 1 \mathrm{mM} \mathrm{MgCl} 2, \mathrm{pH} 7.0$ ), which correspond to a reversible equilibrium shift from a predominantly monomeric species to a mixture of monomeric and dimeric species (Fig. $1 \mathrm{G}, \mathrm{H}$; dimer dissociation constant $\mathrm{K}_{\mathrm{d}}=0.9 \pm 0.1 \mu \mathrm{M}$ ). Signals of the AUG hairpin in 5'-L exhibited uniformly reduced intensities as a function of incubation time, and new signals were observed for the 3'-residues of AUG (A345-A356) (Fig. 1H). Similar NMR spectral changes were observed for an isolated AUG oligo-RNA upon titration with an oligo-U5 fragment (Fig. 1I). The NMR chemical shifts and narrow line widths observed for A345A356 indicate that these residues are unstructured and mobile in the dimeric form of the leader $\left(\left[5^{\prime}-\mathrm{L}\right]_{2}\right)$. No ${ }^{1} \mathrm{H}^{-13} \mathrm{C}$ HMQC signals were detected for G328-G344 of $\left[5^{\prime}-\mathrm{L}\right]_{2}$ due to severe line broadening (discussed below). These findings are consistent with a phylogenetically-derived structural model $(10,20)$, in which the 5'-residues of AUG base pair with U5 and the 3'-residues are disordered (Fig. 1C).

To directly probe for U5:AUG base pairing, we developed an NMR approach that involves replacement of a short stretch of adjacent base pairs by A-U base pairs (lr-AID; long-range probing by Adenosine Interaction Detection). The substituting element (ideally $\left[\mathrm{U}_{\mathrm{i}} \mathrm{U}_{\mathrm{j}} \mathrm{A}_{\mathrm{k}}\right]$ : $\left[\mathrm{U}_{1} \mathrm{~A}_{\mathrm{m}} \mathrm{A}_{\mathrm{n}}\right]$, but other sequences can suffice) (Fig. $2 \mathrm{~A}$ ) affords an upfield-shifted $\mathrm{A}_{\mathrm{m}}-\mathrm{C}_{2}-^{-1} \mathrm{H}$ NMR signal ( $\sim 6.5 \mathrm{ppm}$ ), enabling direct detection of cross-strand $\mathrm{A}_{\mathrm{k}}-\mathrm{H} 2$ and $-\mathrm{H} 1^{\prime}{ }^{1} \mathrm{H}-{ }^{1} \mathrm{H}$ nuclear Overhauser effects (NOEs) without the need for heteronuclear spectral editing. The HIV-1 ${ }_{\text {NL4-3 }}$ leader naturally contains one [UUA:UAA] element in the TAR hairpin ([U12A14]:[U45-A47]), and to preclude signal overlap, A46 was mutated to G (a naturally occurring substitution in 7\% of the reported HIV-1 TAR sequences (31)) (Fig. 2B-D). NOESY spectra (28) obtained for the lr-AID modified dimeric 5'-leader ([5'L $\left.^{\text {lr-AID-U5:AUG }}\right]_{2}$, C110-G112 and G338-G339 substituted by UUA and AA, respectively; Fig. 2A) exhibited well-resolved A338-H2 signals with frequencies similar to those observed for an isolated lr-AID modified U5:AUG oligoribonucleotide (Fig. 2E,F). Other outlier signals in the NOESY spectrum were unaffected by the lr-AID substitutions. All of the expected A338-H2 inter-adenosine NOEs were observed, including cross-strand NOEs 
with A112-H1' and - $\mathrm{H} 2$. The A338- $\mathrm{H} 2 \mathrm{~T}_{1}$ and $\mathrm{T}_{2}{ }^{1} \mathrm{H}$ NMR relaxation rates (8.7 sec and 9.4 msec, respectively) are consistent with restricted rotational motion and indicate that U5:AUG resides within a globular region of the $5^{\prime}-\mathrm{L}$ structure.

The relationship between U5:AUG formation and dimerization was investigated by sitedirected mutagenesis. Mutations in AUG designed to stabilize the hairpin and disrupt base pairing with U5 (5'- $\mathrm{L}^{\mathrm{AUG}-\mathrm{HP}}$ ) favored the monomer, whereas mutations that promote U5:AUG base pairing and destabilize the hairpin $\left(\left[5^{\prime}-\mathrm{L}^{\mathrm{U} 5: \mathrm{AUG}}\right]_{2}\right.$ ) favored the dimer (Fig. $\mathrm{S} 1$ ). Deletion of AUG (5'-L $\mathrm{L}^{\Delta \mathrm{AUG}}$ ) also favored the monomer (Fig. 3A), whereas incubation of $5^{\prime}-\mathrm{L}^{\Delta \mathrm{AUG}}$ with a 17-nucleotide AUG oligoribonucleotide (G328-G344; AUG-17) promoted dimerization (Fig. 3B). These findings indicate that dimerization is suppressed when AUG exists in a hairpin conformation $\left(\mathrm{AUG}^{\mathrm{HP}}\right)$, that $\mathrm{U}$ 5:AUG formation promotes dimerization, and that dimerization is induced by intramolecular U5:AUG base pairing rather than intermolecular tethering.

NMR chemical shifts and NOEs associated with A268-H2 in 5'-L, 5'- $\mathrm{L}^{\Delta \mathrm{AUG}}$, and an isolated DIS oligo-RNA were similar, indicating that the attenuated dimerization activity of the $\mathrm{AUG}^{\mathrm{HP}}$ form of $5^{\prime}-\mathrm{L}$ is not due to re-folding of the DIS (32). Sequence complementarity between U5 and the GC-rich loop of the DIS suggested that dimerization may instead be inhibited by U5:DIS base pairing. Consistent with this hypothesis, mutations in $5^{\prime}$-L designed to enhance U5:DIS base pairing promoted monomer formation (Fig. 3C,D), whereas mutations to disrupt U5:DIS base pairing induced dimerization of $5^{\prime}-\mathrm{L}^{\triangle \mathrm{AUG}}$ (Fig. S2). In addition, NMR data obtained for an lr-AID substituted 5'-L $\mathrm{L}^{\triangle \mathrm{AUG}} \mathrm{RNA}$ with 5'$\mathrm{L}^{\triangle \mathrm{AUG}}$-like dimerization and NC binding properties (Fig. S4) exhibited cross-strand NOEs and NMR chemical shifts consistent with the predicted U5:DIS interface (Fig. 3E-G).

To determine if U5:AUG formation influences NC binding, isothermal titration calorimetry (ITC) experiments were performed with 5'-L and mutant 5' $5^{\prime} \mathrm{L}^{\mathrm{AUG}-\mathrm{HP}}$ and 5'- $\mathrm{L}^{\mathrm{U} 5: \mathrm{AUG}}$ RNAs. Under conditions of the ITC experiments, $5^{\prime}-\mathrm{L}^{\mathrm{AUG}-\mathrm{HP}}$ and $5^{\prime}-\mathrm{L}^{\mathrm{U} 5: A U G}$ exist predominantly as monomers and dimers, respectively, and native $5^{\prime}$-L exists as a 70:30 monomer:dimer equilibrium mixture (Fig. 4A). All three RNAs gave rise to two-component $\mathrm{NC}$ binding isotherms that included an initial exothermic event associated with high-affinity NC binding, and a subsequent endothermic event attributed to NC-induced RNA unfolding at high NC:RNA ratios (33) (Fig. 4B and Fig. S3). Whereas 5'-L $5^{\text {AUG-HP }}$ binds $7 \pm 1$ NC molecules with high affinity $\left(\mathrm{K}_{\mathrm{d}}=87 \pm 3 \mathrm{nM}\right),\left[5^{\prime}-\mathrm{L}^{\mathrm{U} 5: \mathrm{AUG}}\right]_{2}$ binds $32 \pm 2 \mathrm{NC}$ molecules with high affinity $\left(\mathrm{K}_{\mathrm{d}}=71 \pm 3 \mathrm{nM}\right)\left(\mathrm{Fig}\right.$. 4B). The ITC profile observed for native $5^{\prime}-\mathrm{L}$ was similar to that obtained for a 70:30 mixture of $5^{\prime}-\mathrm{L}^{\mathrm{AUG}-\mathrm{HP}}$ and $5^{\prime}-\mathrm{L}^{\mathrm{U} 5: \mathrm{AUG}}$ (Fig. 4A,B), validating the use of $5^{\prime}-\mathrm{L}^{\mathrm{AUG}-\mathrm{HP}}$ and $5^{\prime}-\mathrm{L}^{\mathrm{U}} \mathrm{A}: \mathrm{AUG}$ as models for the native monomer and dimer, respectively. Importantly, the NC binding and dimerization properties of $5^{\prime}-$

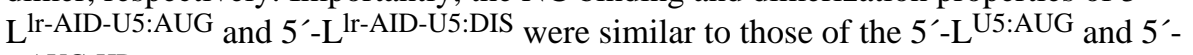
$\mathrm{L}^{\text {AUG-HP }}$ controls, respectively (Fig. S4), further indicating that the lr-AID substitutions did not alter the structure of the RNA. Substitution of the dimer-promoting GC-rich loop of the DIS hairpin by a GAGA tetraloop prevented dimerization of $5^{\prime}-\mathrm{L}^{\mathrm{U} 5: \mathrm{AUG}}$ but did not affect its NC binding properties (Fig. S5), indicating that enhanced NC binding by the U5:AUG form of the 5'-leader is due to intramolecular conformational changes associated with U5:AUG base pairing and not to dimerization per se.

The above findings suggested that in vivo RNA packaging should be dependent on U5:AUG formation, and we therefore measured packaging efficiencies of vector RNAs containing 5'$\mathrm{L}^{\mathrm{AUG}-\mathrm{HP}}$ and 5'- $\mathrm{L}^{\mathrm{U} 5: \mathrm{AUG}}$ mutations in competition experiments. An HIV-1 ${ }_{\mathrm{NL4}-3}$ helper construct that expressed the native 5'-leader and all viral proteins except Env was co-

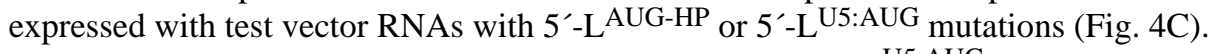
Consistent with the structural and NC binding studies, the 5'- $\mathrm{L}^{\mathrm{U} 5: \mathrm{AUG}} \mathrm{RNAs}$ were packaged 
nearly as avidly as the native construct (Fig. 4C; lane 5) whereas the $5^{\prime}-\mathrm{L}^{\text {AUG-HP }}$ RNAs exhibited severe packaging defects (Fig. 4C; lane 6).

Previously observed packaging defects associated with mutations in AUG $(24,34)$ can be attributed to defects in U5:AUG dependent exposure of NC binding sites rather than inhibition of a cis-packaging mechanism (34). The inability of helper RNAs to rescue packaging of RNAs with AUG mutations (24) is likely due to a defect in U5:AUG dependent exposure of the DIS since rescue requires DIS-mediated heterodimer formation. The relationship between U5:AUG formation and dimerization also explains why mutations in AUG can lead to the production of virions containing genomes that are sensitive to dissociation by mild denaturants (25). Hybrid 5'-leader structures containing both U5:AUG and $\mathrm{AUG}^{\mathrm{HP}}$ features, predicted from in vivo ribose reactivity measurements (22), were not observed by NMR but can be explained by the presence of the $\mathrm{AUG}^{\mathrm{HP}} / \mathrm{U} 5: \mathrm{AUG}$ equilibrium. Elevated U5 nucleotide reactivities observed by traditional chemical probing, which are incompatible with an exclusive U5:AUG structure (21), may also be explained by the observed $\mathrm{AUG}^{\mathrm{HP}} / \mathrm{U} 5$ :AUG equilibrium. The finding that the gag start codon is exposed in a mobile hairpin in the monomeric leader and sequestered in the dimer is consistent with observations that dimerization attenuates both the chemical reactivity of the gag start codon and the in vitro translational activity of the genome (16).

In summary, we have developed an approach that extends the size of RNAs that can be structurally probed by NMR to $\sim 230$ kDaltons. The 1r-AID method avoids NMR relaxation problems associated with heteronuclear editing, probes interactions over a narrow distance range ( $\leq \sim 5 \AA$ ), provides both chemical shift and distance information for structural analysis, and is readily implemented using standard 2D NMR experiments and conservative base pair mutagenesis. Our findings support a translation/packaging RNA structural switch mechanism, in which the dimer-promoting GC-rich loop of the DIS hairpin is sequestered by base pairing with $\mathrm{U} 5$ in the $\mathrm{AUG}^{\mathrm{HP}}$ form of the 5'-leader, and is displaced by AUG upon U5:AUG formation (Fig. 4D). Conformational changes associated with U5:AUG base pairing simultaneously sequester the gag start codon and expose the DIS and high affinity $\mathrm{NC}$ binding sites, thereby attenuating translation and promoting the packaging of a dimeric genome.

\section{Supplementary Material}

Refer to Web version on PubMed Central for supplementary material.

\section{Acknowledgments}

This research was supported by a grant from the National Institute of General Medical Sciences (NIGMS; R01 GM42561). L.G. was supported by an NIGMS grant for maximizing doctoral diversity (R25 MBRS-IMSD GM55036). B.D., K.E., S.J., G.K. and S.B. were supported by an NIGMS grant for enhancing minority access to research careers (MARC U*STAR 2T34 GM008663). B.D., G.K. and S.B. were supported by an HHMI undergraduate education grant. We thank Steven R. King (Michigan) and HHMI staff (UMBC) for technical assistance.

\section{References}

1. Coffin, JM.; Hughes, SH.; Varmus, HE. Retroviruses. Plainview, N.Y.: Cold Spring Harbor Laboratory Press; 1997.

2. Lever AM. Adv Pharmacol. 2007; 55

3. Onafuwa-Nuga A, Telesnitsky A. Microbiol Mol Biol Rev. 2009; 73

4. Poole E, Strappe P, Mok HP, Hicks R, Lever AM. Traffic. 2005; 6

5. Moore MD, et al. PLoS Pathog. 2009; 5 
6. Kutluay SB, Bieniasz PD. PLoS pathogens. 2010; 6

7. Paillart J-C, Shehu-Xhilaga M, Marquet R, Mak J. Nature Revs. Microbiol. 2004; 2

8. Jouvenet N, Simon SM, Bieniasz PD. Proc. Natl. Acad. Sci. USA. 2009; 106

9. Miele G, Mouland A, Harrison GP, Cohen E, Lever AM. J. Virol. 1996; 70

10. Abbink TEM, Berkhout B. J. Biol. Chem. 2003; 278

11. Russell RS, Liang C, Wainberg MA. Retrovirology. 2004; 1

12. Greatorex J. Retrovirology. 2004; 1

13. D'Souza V, Summers MF. Nature Revs. Microbiol. 2005; 3

14. Lu K, Heng X, Summers MF. J. Mol. Biol. 2011; 410

15. Harrison GP, Lever AML. J. Virol. 1992; 66

16. Baudin F, et al. J. Mol. Biol. 1993; 229

17. Clever J, Sassetti C, Parslow TG. J. Virol. 1995; 69

18. McBride MS, Panganiban AT. J. Virol. 1996; 70

19. Clever JL, Miranda J, D, Parslow TG. J. Virol. 2002; 76

20. Damgaard CK, Andersen ES, Knudsen B, Gorodkin J, Kjems J. J. Mol. Biol. 2004; 336

21. Paillart JC, et al. J. Biol. Chem. 2004; 279

22. Wilkinson KA, et al. PLoS Biology. 2008; 6

23. Watts JM, et al. Nature. 2009; 460

24. Nikolaitchik O, Rhodes TD, Ott D, Hu W-S. J. Virol. 2006; 80

25. Song R, Kafaie J, Laughrea M. Biochemistry. 2008; 47

26. Allain FH-T, Varani G. J. Mol. Biol. 1997; 267

27. Tolbert BS, et al. J. Biomol. NMR. 2010; 47

28. Materials and methods are available as supporting material.

29. Kerwood DJ, Cavaluzzi MJ, Borer PN. Biochemistry. 2001; 40

30. Yu ET, Hawkins A, Eaton J, Fabris D. Proc. Natl. Acad. Sci. USA. 2008; 105

31. The HIV Sequence Database. 2011. <http://www.hiv.lanl.gov/content/sequence/NEW-ALIGN/ align.html>

32. Huthoff H, Berkhout B. RNA. 2001; 7

33. Summers MF, et al. Protein Sci. 1992; 1

34. Poon DT, Chertova EN, Ott DE. Virology. 2002; 293 
A
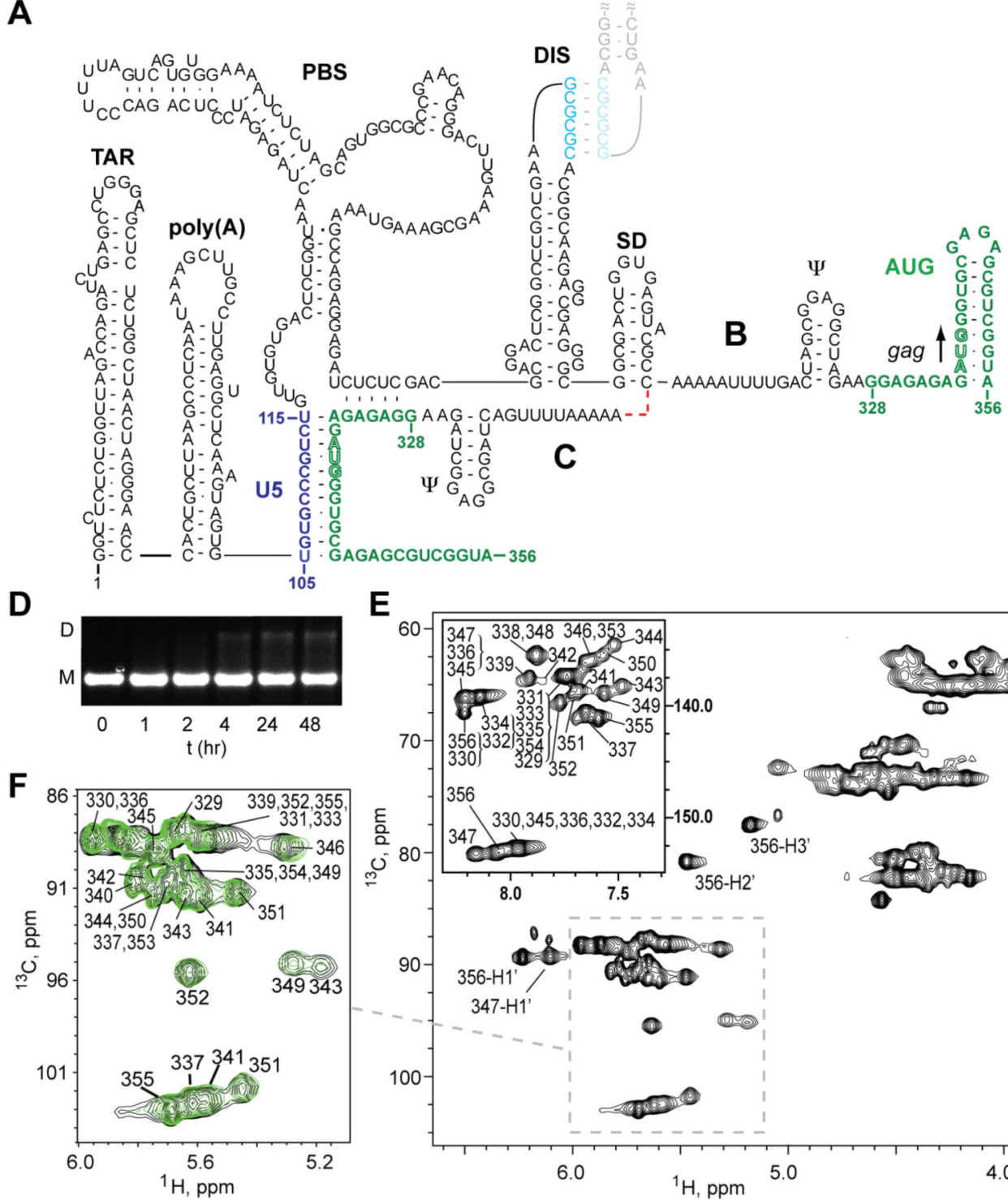

C
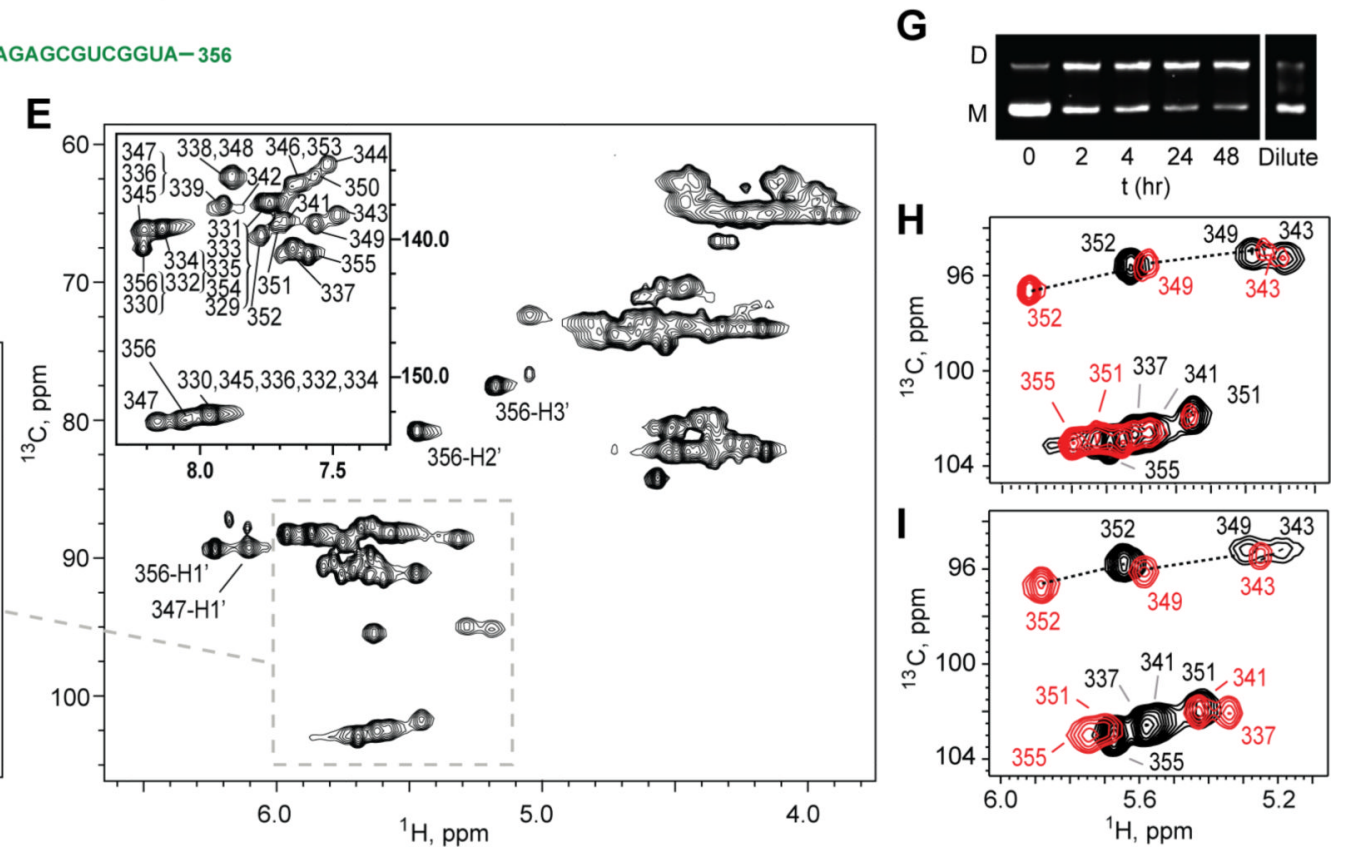

Fig. 1. Structure of the HIV-1 5'-leader

(A-C), Secondary structure predictions showing AUG (green) in hairpin (B) and U5:AUG

(C) conformations. (D) 5'-L forms a monomer at low ionic strength (LI buffer $=10 \mathrm{mM}$

Tris-HCl, $10 \mathrm{mM} \mathrm{NaCl}, \mathrm{pH} 7.0)([\mathrm{RNA}]=60 \mu \mathrm{M})$. (E) $2 \mathrm{D}{ }^{1} \mathrm{H}-{ }^{13} \mathrm{C}$ HMQC NMR spectrum obtained for $5^{\prime}$-L containing ${ }^{13} \mathrm{C}$-labeled AUG (5'-L $5^{-\mathrm{AUG}^{*}}$; LI buffer). (F) Overlay of spectra

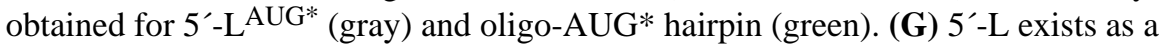
reversible monomer-dimer equilibrium in PI buffer ([5'-L] $=30 \mu \mathrm{M}$ in left panel). The equilibrium shifts to the monomer upon subsequent dilution (100-fold) in PI buffer.

(H) ${ }^{1} \mathrm{H}_{-}{ }^{13} \mathrm{C}$ HMQC NMR data obtained for $5^{\prime}-\mathrm{L}^{\mathrm{AUG}}$ in LI buffer (black) and immediately upon dissolution in PI buffer (red). Spectral changes correlate with an equilibrium shift toward the dimer. Residues 349, 351, 352 and 355 are unstructured and detectable, and residues 337 and 341 are rotationally restricted and undetectable, in the $\left[5^{\prime}-\mathrm{L}\right]_{2}$ dimer. (I) Similar spectral changes were observed upon titration of an isolated $\mathrm{AUG}^{*}$ hairpin (black) with an unlabeled U5 oligonucleotide, except that all U5:AUG signals were detectable (red). 
A

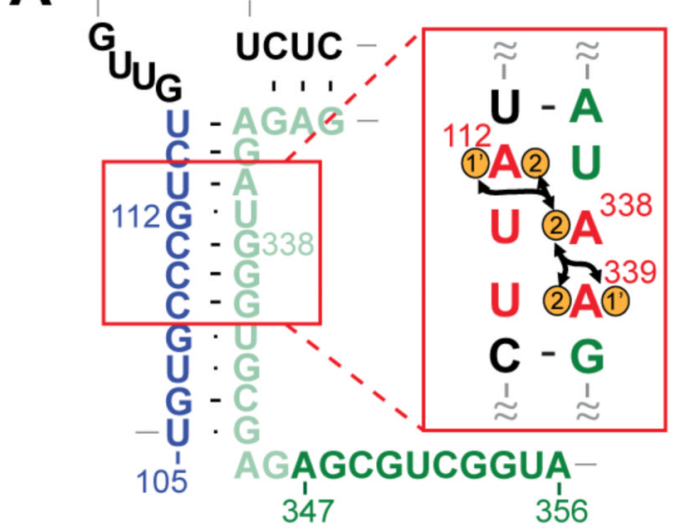

B

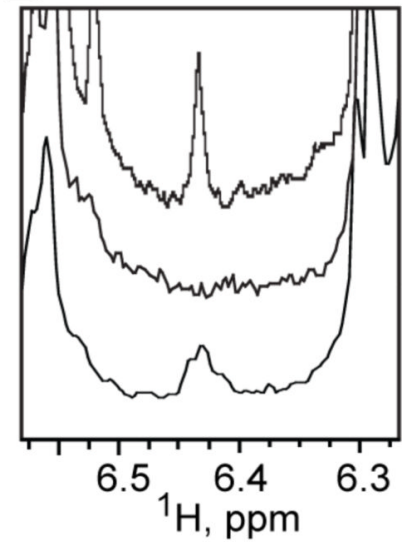

C

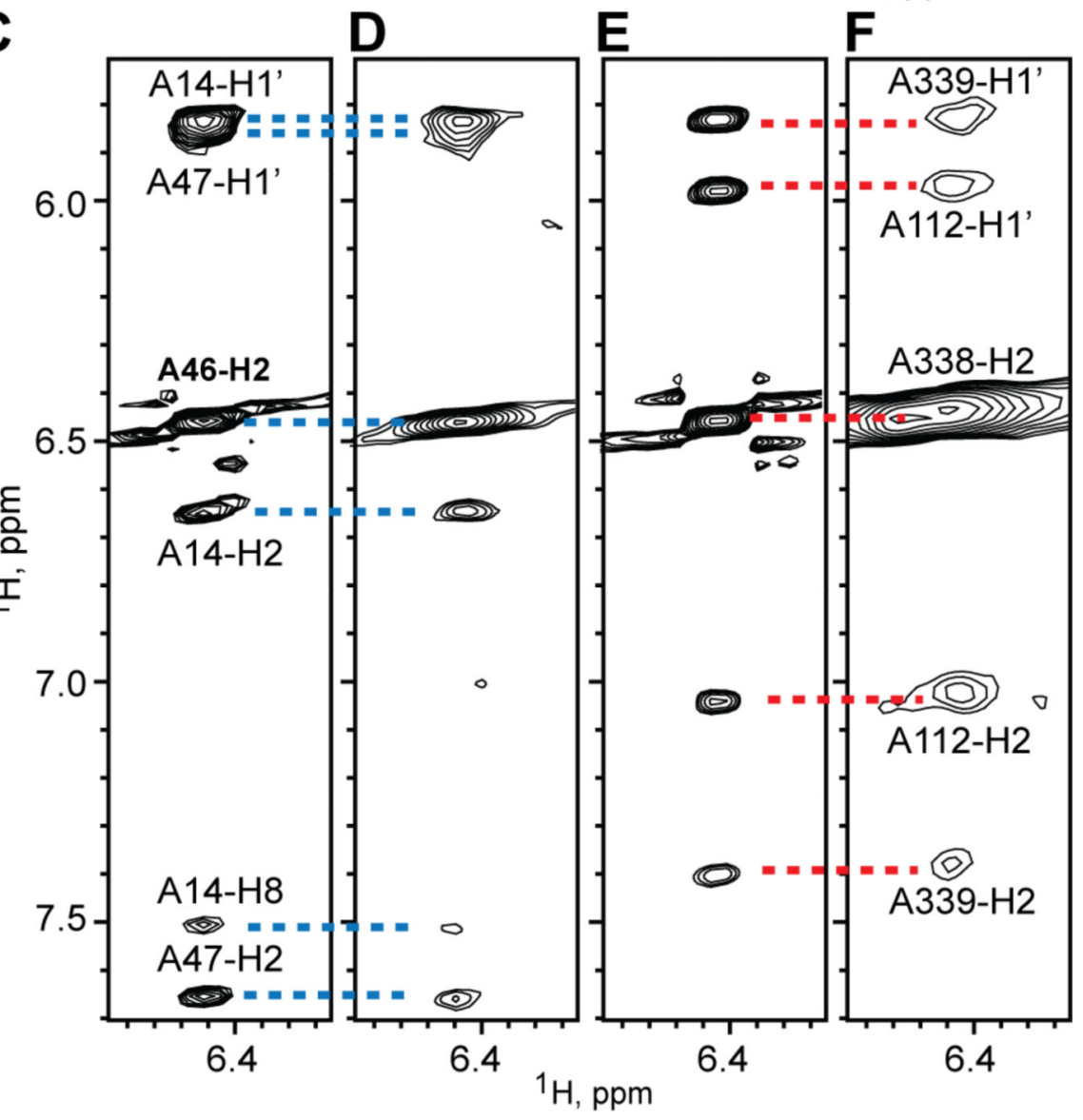

Fig. 2. AUG base pairs with U5 in the dimer

(A) lr-AID mutations (red) designed to probe for predicted U5:AUG base pairing. Black arrows denote ${ }^{1} \mathrm{H}-{ }^{1} \mathrm{H}$ NOEs. (B) Portions of $1 \mathrm{D}^{1} \mathrm{H}$ NMR spectra showing (top): the TAR A46-H2 signal of native $\left[5^{\prime}-\mathrm{L}\right]_{2}$; (middle): A46G substitution ([5'- $\left.\mathrm{L}^{\mathrm{A} 46 \mathrm{G}}\right]_{2}$ ) eliminates the TAR A46-H2 signal; (bottom): A338-H2 signal observed for lr-AID substituted [5'$\left.\mathrm{L}^{\mathrm{A} 46 \mathrm{G}}\right]_{2}$. (C,D) Similarities in NOE spectra observed for A46-H2 of an isolated TAR oligonucleotide (C) and native $\left[5^{\prime}-\mathrm{L}\right]_{2}$ (D). (E,F) Similar A338-H2 NOEs observed for an isolated lr-AID U5:AUG oligonucleotide (E) and lr-AID substituted [5'- $\left.\mathrm{L}^{\mathrm{A} 46 \mathrm{G}}\right]_{2}(\mathbf{F})$. 
A

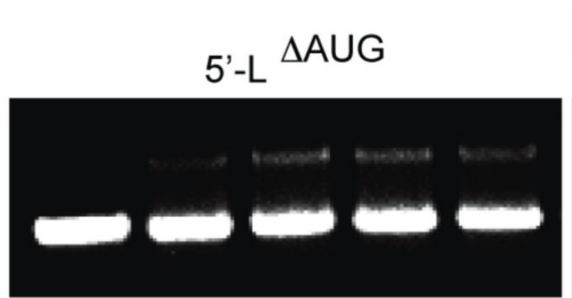

C

$\begin{array}{lllll}0 & 1 & 24 & 32 & 48\end{array}$

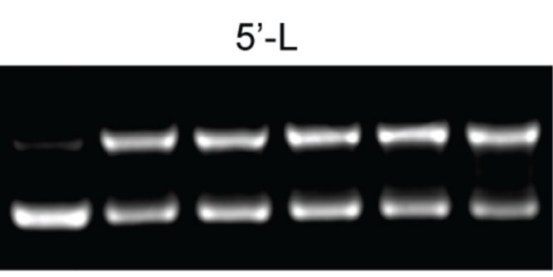

$\begin{array}{llllll}0 & 1 & 2 & 4 & 24 & 48\end{array}$

$\mathbf{E}$

$\mathbf{F}$

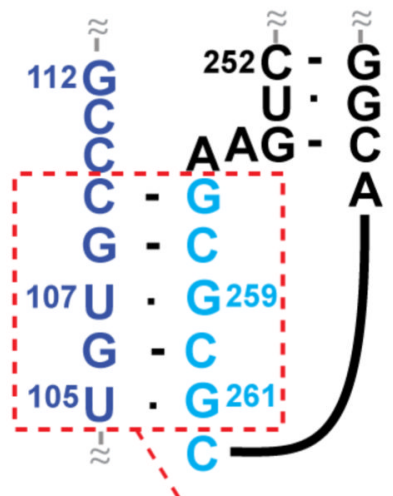

$109 \mathrm{U}-\mathrm{A}^{257}$

(1)A(2) U

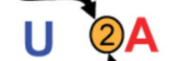

U (2)A(1)

$105 C-G 261$

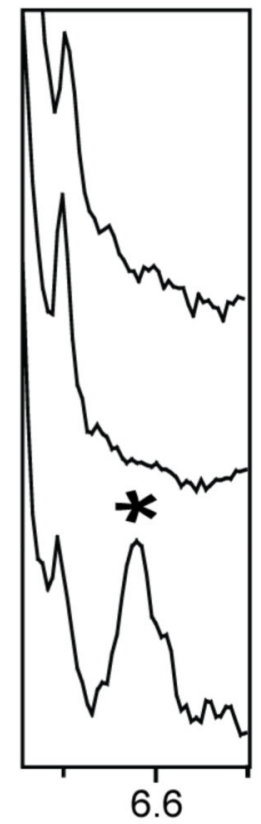

${ }^{1} \mathrm{H}, \mathrm{ppm}$
B 5'-L $\triangle A U G+A U G-17$

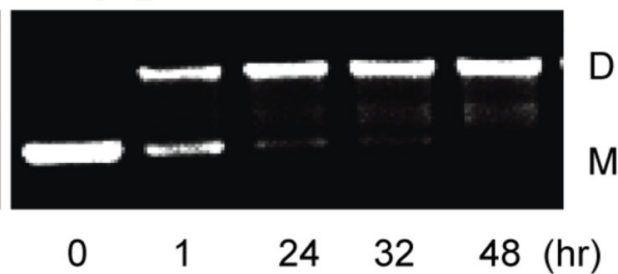

D 5'-L U105C

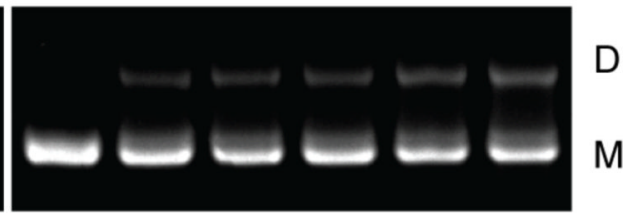

$\begin{array}{lllll}1 & 2 & 4 & 24 & 48\end{array}(\mathrm{hr})$

G

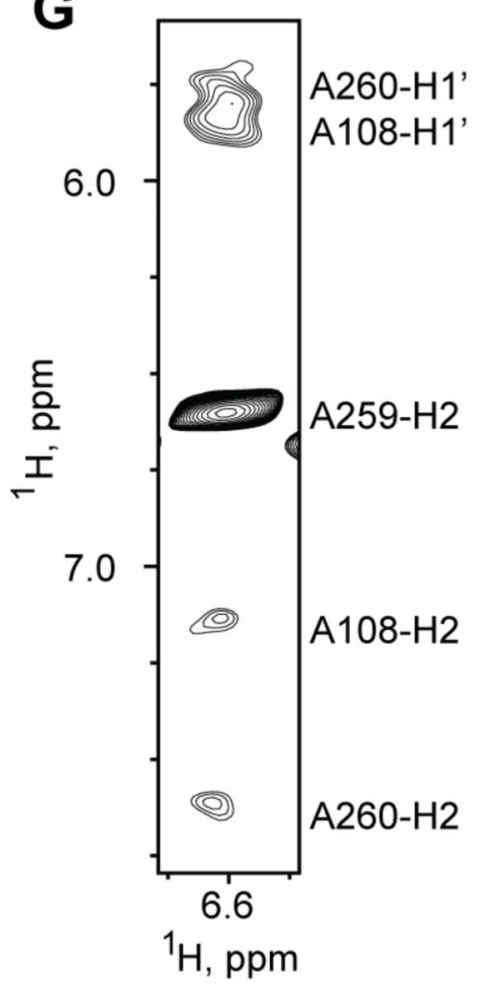

Fig. 3. U5:AUG formation promotes dimerization

(A) $5^{\prime}-\mathrm{L}^{\Delta \mathrm{AUG}}(1 \mu \mathrm{M})$ forms a stable monomer. (B) Addition of AUG-17 (10-fold molar excess) promotes dimerization of $5^{\prime}-\mathrm{L}^{\Delta \mathrm{AUG}}$. (C,D) Substitutions that enhance U5:DIS interactions favor the monomer. (E) Proposed U5:DIS base pairing and lr-AID sequences used for structural probing. (F) The A259-H2 signal is only observed when U5 and DIS both contain lr-AID substitutions. Top: $5^{\prime}-\mathrm{L}^{\Delta \mathrm{AUG}}$, A46G . Middle: $5^{\prime}-\mathrm{L}^{\Delta \mathrm{AUG}}{ }^{\mathrm{A}} 46 \mathrm{G}$ containing the lr-AID substitution in the DIS loop only. Bottom: $5^{\prime}-\mathrm{L}^{\Delta \mathrm{AUG}, \mathrm{A} 46 \mathrm{G}}$ containing $1 \mathrm{r}-\mathrm{AID}$ substitution in both DIS and U5. (G) 2D NOE data for A259-H2 in 1r-AID substituted 5'$\mathrm{L}^{\triangle \mathrm{AUG}} \mathrm{A} 46 \mathrm{G}$, assigned from the corresponding U5:DIS oligonucleotide control spectrum. 


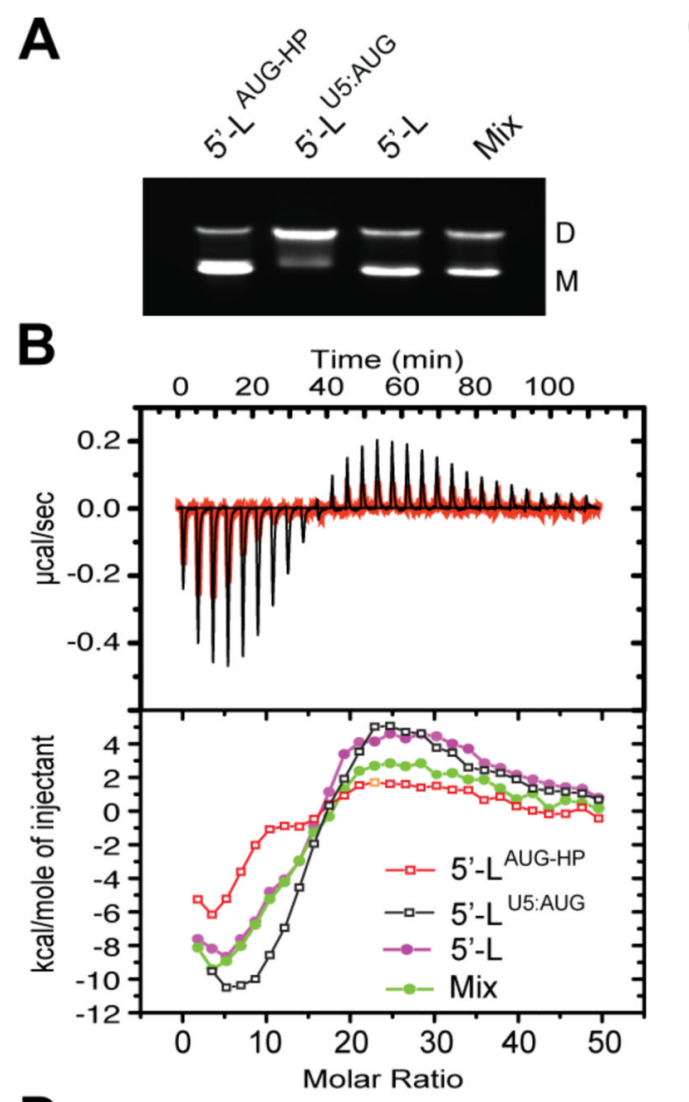

C

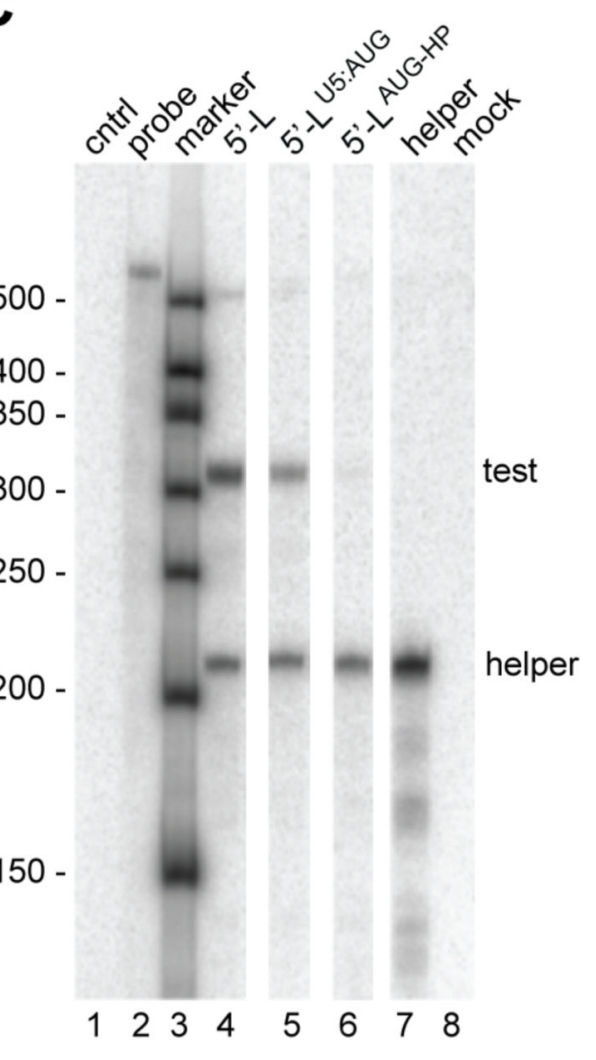

D
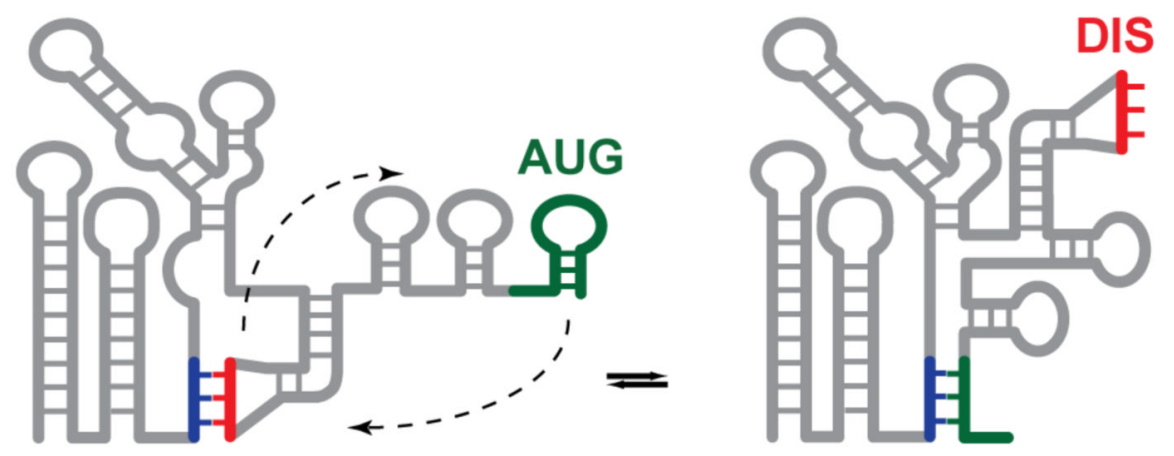

U5 DIS

U5 AUG

Promotes Translation

Promotes Dimerization, NC Binding, Packaging

Fig. 4. U5:AUG formation promotes NC binding and packaging

(A) 5'-L AUG-HP and 5'-L $5^{\mathrm{U}}: \mathrm{AUG}$ form predominantly monomers and dimers, respectively, and native $5^{\prime}$-L adopts a 70:30 monomer:dimer equilibrium (PI buffer; [RNA] $=0.8 \mu \mathrm{M}$ ). MIX = 70:30 mixture of 5'-L AUG-HP and 5'-L U5:AUG . (B) ITC NC titration data for samples shown in (A). (C) Packaging of native HIV-1 ${ }_{N L 4-3} 5^{\prime}-\mathrm{L}, 5^{\prime}-\mathrm{L}^{\mathrm{U} 5: A U G}$ and 5'-L AUG-HP RNAs under competition conditions (assayed by RNase protection; see Supplementary Methods). Lanes 2-8: 2: undigested probe; 3 : RNA size standards; 4: native $\mathrm{HIV}-1_{\mathrm{NL} 4-3}$ helper versus native HIV-1 ${ }_{\mathrm{NL} 4-3}$ test vector RNAs; 5 : native HIV-1 ${ }_{\mathrm{NL} 4-3}$ helper versus $5^{\prime}-\mathrm{L}^{\mathrm{U} 5: \mathrm{AUG}}$ test vector; 6: native HIV-1 $1_{\mathrm{NL} 4-3}$ helper versus $5^{\prime}-\mathrm{L}^{\mathrm{AUG}-\mathrm{HP}}$ test vector; 7 : HIV-1 ${ }_{\mathrm{NL} 4-3}$ helper 
expressed without test RNA; 8: mock transfected-cells. Bands corresponding to native HIV-1 NL4-3 helper RNA (helper) and co-packaged test RNAs (test) are labeled. (D) Nucleotide displacement mechanism regulates dimeric genome packaging. 